\title{
ERRATUM
}

\section{DNA Barcoding for Industrial Quality Assurance}

Tiziana Sgamma, Claire Lockie-Williams, Marco Kreuzer, Sarah Williams, Ulrike Scheyhing, Egon Koch, Adrian Slater, Caroline Howard

Planta Med, advance online publication June 29, 2017

Planta Med 2017; 83: 1117-1129

DOI: $10.1055 / \mathrm{s}-0043-113448$

The following Acknowledgements have been added: Marco Kreuzer was supported by MEDPLANT, a Marie Curie Actions Initial Training Network (ITN), funded by the European Union under the Seventh Framework Program. 